\title{
Harmonic Plane Decomposition: An Extension of the Vector-Space Decomposition - Part II
}

\author{
Gustaf Falk Olson*, Yixuan $\mathrm{Wu}^{\dagger}$, Luca Peretti ${ }^{\ddagger}$, Oskar Wallmark ${ }^{\S}$ \\ Division of Electric Power and Energy Systems \\ KTH Royal Institute of Technology \\ Stockholm, Sweden \\ * gufo@kth.se† yixuanw@kth.se $\ddagger$ lucap@kth.se $§$ owa@kth.se
}

\begin{abstract}
In this paper the harmonic plane decomposition from part $I$ is completed. Multiphase electrical machines with independently controlled stator coils benefit from a fixed amount of current controllers and unified models. In part I, the foundation for such models was laid. This paper shows that the choice of the base case has to fulfill certain criteria to properly represent vector-space quantities. More specifically, phase-pole configurations with an even number of pole pairs need adapted base cases with reduced rank as compared to the odd base case. Continuous modeling and control requires retention of the dimensionality of Clarke matrices. A different number of stator and rotor slots can lead to aliasing in the transformation, when keeping the dimension consistent. Therefore, a specific Clarke transformation for rotor quantities based on the developed theory is elaborated. Finally, it is demonstrated how the harmonic plane decomposition can be used to model each harmonic plane of an arbitrary phase-pole configuration with conventional machine equivalent circuits, e.g. the T-equivalent or inverse- $\Gamma$ models.

Index Terms-complex winding factor, DFT, harmonic plane decomposition, multiphase electric machines, vector-space decomposition
\end{abstract}

\section{INTRODUCTION}

This paper is the final contribution to a two-part series introducing a harmonic plane decomposition (HPD). The vectorspace decomposition (VSD) as described in [1] provides a theoretical framework to properly describe fundamental quantities (currents, voltages, and flux linkages) and their harmonics in multiphase electrical machines (MPEMs). It aims to map these machine quantities into orthogonal vector spaces, where the conventional vector-oriented control algorithms of three-phase machines are applicable [2]. One drawback is that the number of vector spaces increases with the number of phases, $m$. In fact, the number of vector spaces equals $\lceil\mathrm{m} / 2\rceil$. This is expected to pose a problem when controlling multiphase electrical machines with phase-changing capabilities, e.g. YY30 machines [3], since the number of controllers must change with the number of phases. Another drawback is that the transformation assumes $m$ fixed magnetic axes. This, in turn, is a problem because it assumes that all currents in a phase belt are the same. This can only be guaranteed if the conductors in the slots belonging to the same phase belt are series-connected. If not, such as in [4]-[6], the VSD does not offer the possibility to actively control the currents in adjacent slots belonging to the same phase belt.
In [3] information from all $Q_{s}$ stator slots was used to transform space-vector quantities into equivalent fixed $(\alpha \beta)$ and rotating $(d q)$ referenced frames. A base case with $m_{b}=Q_{s} / 2$ was introduced to represent all possible machine configurations. It was shown that the HPD is nothing but a discrete Fourier transformation (DFT) in the space domain of the space-vector quantities of each slot.

The method was exemplified for a machine with $Q_{s}=36$ configured as either an $m=3$ or $m=6$ phase and $p=2$ pole pair machine. The benefit of this approach is that statorcoil reconfigurable machines such as those in [4]-[6] can be represented in a uniform manner. In practice, it means that the amount of current controllers in a real setup remains the same, independently of the number of poles and phases, and that the entire machine can be correctly modeled using lumped parameters with a fixed number of equivalent circuits. Moreover, the dynamic transition between two different phasepole configurations can be seamlessly controlled.

Part II in this series adds three important findings. Firstly, it treats cases when the pole-pair number, $p$, is even. The matrices obtained from the VSD and HPD presented in [3] consider only odd-order harmonics. Therefore, a phasepole configuration resulting in even-order space harmonics in mechanical angles cannot be represented with a base case of $m_{b}=Q_{s} / 2$. The reason for this and how to choose an appropriate base case while retaining the dimensions of the original base case, is explained in Section II. Secondly, Section III focuses on the transformations of rotor quantities into stator-equivalent quantities necessary for the machine modeling. Thirdly, both of these sections deal with the problem of aliasing, which inevitably occurs when the base case is changed and whenever $Q_{r} \neq Q_{s}$. Section IV concludes with simulations showing the applicability of the presented results.

Finally, Section V features a research outlook and Section VI summarizes the findings.

\section{Even Base CASES}

In this section, the necessity of additional base cases for even numbers of pole pairs is explained. This is followed by the introduction of the adapted Clarke transformation matrix for the even base cases. Nevertheless, all characteristics of the odd base case are preserved, not least the dimension of the matrices. 


\section{A. Motivation}

Both the standard and extended Clarke transformations contain odd harmonics only. Therefore, only odd harmonic planes exist [3]. This is in accordance to the outcome of the DFT for a periodic and half-wave symmetric signal, e.g. the current distribution in the slots over an odd number of pole-pairs. It is known that the Fourier transformation of any L-periodic signal, $f(x)$, with half-wave symmetry, $\left(f\left(x-\frac{L}{2}\right)=-f(x)\right)$, contains only odd harmonics. Recalling that the extended Clarke transformation is indeed a DFT in space domain, as shown in [3], the existence of odd harmonics only infers that it is a necessary condition for the space-vector quantity to be half-wave symmetric for the Clarke transformation to be functional.

However, a variable phase-pole electrical machine, e.g. the wound independently-controlled stator coils machine (WICSC machine) in [4], can be operated with odd as well as even pole-pair numbers. In case the machine is operated with an even number of pole pairs, only even harmonics appear in the frequency domain with respect to the mechanical angles. Hence, with the extended Clarke transformation introduced in [3], these cannot be resolved.

The extended Clarke transformation matrix on the one side performs a Fourier analysis when transforming from the 123 fundamental reference frame to the $\alpha \beta$ reference-frame. On the other side, it performs a Fourier synthesis when taking the reverse transformation. The odd base case described in [3] has $p_{b}=1$ pole pairs and the current distribution is sampled $Q_{s}$ times within one space-fundamental period, where $Q_{s}$ is the number of stator slots. The selection of $p_{b}$ and the number of sampling points have two important implications.

Firstly, the current distribution in the slots of a machine with an even pole-pair number repeats over $Q_{s} / p$ slots. Therefore, it does not possess half-wave symmetry over the full $Q_{s}$ slots ( $360^{\circ}$ mechanically) sampling length. As described above, half-wave symmetry is necessary for the Clarke transformation to correctly represent the current distribution. Consequently, (an)other base-case(s) need(s) to be implemented to obtain such a half-wave symmetry. Formally, (1) states a necessary condition to achieve half-wave symmetry:

$$
\frac{\pi / p_{b}}{\pi / p}=\frac{p}{p_{b}}=2 n+1, \quad n \in\{0,1,2, \ldots\}
$$

Note that any machine with an odd pole-pair number, i.e. $p \in\{1,3,5, \ldots\}$, will be half-wave symmetric when $p_{b}=1$ is chosen. On the contrary, $p_{b}$ must be selected with more care for even pole-pair machines. In the following $m_{b, o}$ and $m_{b, e}$ denote the number of phases for the odd and even base cases respectively. In the same manner $p_{b, o}$ and $p_{b, e}$ denote the number of pole pairs for the odd and even base cases.

Secondly, according to the Nyquist-Shannon sampling theorem, space-harmonic orders up to $m_{b}=Q_{s} /\left(2 p_{b}\right)$ can be resolved by a DFT with $Q_{s}$ unique samples. The situation for the odd base case $m_{b, o}=Q_{s} /\left(2 p_{b, o}\right)$ is depicted in Fig. 1a. The Nyquist-Shannon window is highlighted in light gray. The

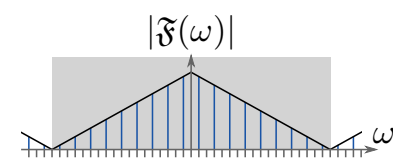

(a) Correct HPD without aliasing.

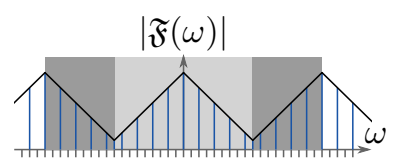

(b) Erroneous HPD with aliasing.
Fig. 1. DFT of generic signals in frequency domain. The Nyquist-Shannon window is highlighted in light gray. When the Fourier synthesis uses more frequencies than samples per fundamental period, aliasing appears due to the frequency components in the dark gray areas.

number of frequency components in this window corresponds to the rank of the Clarke transformation. Thus, the Fourier synthesis ( $\alpha \beta$-to-123 transformation) is performed correctly. However, only $m_{b, e}=Q_{s} /\left(2 \cdot p_{b, e}\right)<Q_{s}$ unique samples exist per fundamental period of the current distribution in even base cases. In other words, the current distribution wave-form has a higher frequency than in a $p_{b, o}=1$ case, whereas the sampling frequency, $Q_{s}$ samples per $360^{\circ}$ mechanically, remains the same. Hence, fewer frequency components can be resolved than in the $p_{b, o}=1$ case. This is indicated in Fig. $1 \mathrm{~b}$ by the smaller Nyquist-Shannon window in light gray. However, when applying the extended Clarke transformationmatrix for the Fourier synthesis ( $\alpha \beta$-to-123 transformation) with maintained rank, frequencies outside of the NyquistShannon limit, i.e. in the dark gray areas, are taken into account, which results in aliasing.

\section{B. Extended Clarke Transformation Matrix for an Even Base Case}

To resolve even harmonics, a new base case with an even number of pole pairs has to be found. In the same manner as in [3], the phase-pole configuration representing the maximum number of magnetic axes is chosen, i.e. $m_{b, e}=m_{b, o} / k$ phases where $k=p_{b, e} / p_{b, o}$ and the minimum $p_{b, e}$ number of poles is chosen such that (1) is fulfilled. The construction of the transformation matrix stays unchanged as in [3, (2)], with an exception that $\delta_{e}=k \cdot \delta_{o}$ to account for the fact that the current distribution repeats $k$ times over the full space angle $2 \pi$. This results in a matrix $\mathbf{C}_{e}$ analogously to $\mathbf{C}$ in [3] of size $m_{b, e} \times m_{b, e}$ as stated in (2). Here, $\xi$ denotes the biggest odd number, which is smaller than $m_{b, e}$.

$$
\begin{aligned}
\mathbf{C}_{e} & =\left[\begin{array}{ccccc}
1 & \cos (\delta) & \cos (2 \delta) & \ldots & \cos \left(\left(m_{b, e}-1\right) \delta\right) \\
0 & \sin (\delta) & \sin (2 \delta) & \ldots & \sin \left(\left(m_{b, e}-1\right) \delta\right) \\
1 & \cos (3 \delta) & \cos (6 \delta) & \ldots & \cos \left(\left(m_{b, e}-1\right) 3 \delta\right) \\
0 & \sin (3 \delta) & \sin (6 \delta) & \ldots & \sin \left(\left(m_{b, e}-1\right) 3 \delta\right) \\
\vdots & \vdots & \vdots & \ddots & \vdots \\
1 & \cos (\xi \delta) & \cos (2 \xi \delta) & \ldots & \cos \left(\left(m_{b, e}-1\right) \xi \delta\right) \\
0 & \sin (\xi \delta) & \sin (2 \xi \delta) & \ldots & \sin \left(\left(m_{b, e}-1\right) \xi \delta\right)
\end{array}\right] \\
\delta & =\delta_{e}=k \cdot \delta_{o}=k \cdot \frac{\pi}{m_{b, o}}=\frac{\pi}{m_{b, e}}
\end{aligned}
$$

To achieve a continuous model, the dimension of the transformation matrices has to be maintained with respect to 
the larger base case $p_{b, o}=1, m_{b, o}=Q_{s} / 2$ as defined by the extended Clarke transformation in [3]. It can be done by expanding $\mathbf{C}_{e}$ to a Clarke transformation matrix $\mathbf{C}_{b i g}$ as constructed in (3). Here, $\mathbf{C}_{e}$ repeats $k$ times in general and $\mathbf{0}$ is a null matrix such that $\operatorname{dim}\left(\mathbf{C}_{b i g}\right)=m_{b, o} \times m_{b, o}$. Recall that $\mathbf{K}_{d}$ is a diagonal matrix containing the winding factors given in $[3,(9)]$.

$$
\begin{aligned}
\mathbf{x}_{\alpha \beta} & =\underbrace{\left(\frac{2}{m_{b, o}}\right)^{K} \mathbf{K}_{d} \mathbf{C}_{b i g}}_{\mathbf{T}_{123 \rightarrow \alpha \beta}} \cdot \mathbf{x}_{123} \\
\mathbf{x}_{123} & =\underbrace{\left(\frac{2}{m_{b, o}}\right)^{1-K} \mathbf{C}_{b i g}^{\mathrm{T}} \mathbf{K}_{d}}_{k \text { times }} \cdot \mathbf{x}_{\alpha \beta} \\
\mathbf{C}_{b i g} & =\underbrace{\left[\begin{array}{cccc}
\mathbf{T}_{\alpha \beta \rightarrow 123} & \mathbf{C}_{e} & \ldots & \mathbf{C}_{e} \\
\mathbf{0} & \mathbf{0} & \ldots & \mathbf{0}
\end{array}\right]}
\end{aligned}
$$

The described approach takes all magnetic axes of the odd base case into account. When the Clarke transformation $\mathbf{T}_{123 \rightarrow \alpha \beta}$ is applied, i.e. performing the Fourier analysis, sampling multiple periods of the $\pi / p_{b, e}$-periodic current does not affect the maximum resolvable frequency component. However, when performing the inverse Clarke transformation, $\mathbf{T}_{\alpha \beta \rightarrow 123}$, i.e. performing the Fourier synthesis, the currents in all coils are reconstructed from the $\alpha \beta$-currents instead of the currents in the slots making up merely one pole. By constructing the $\mathbf{C}_{b i g}$-matrix as in (3), aliased frequency components contained in the last $Q_{s}\left(1-\frac{1}{p_{b, e}}\right)$ elements of the $\alpha \beta$ signal-vector are forced to zero without disregarding these dimensions. Graphically, the aliased frequencies are highlighted in Fig. 1b by the dark gray area. The remaining low order components are within the Nyquist-Shannon window indicated by the light gray area.

\section{Rotor QuANTITIES}

To obtain a full model of the electrical machine, the rotor quantities, i.e. rotor bar currents and rotor flux linkages, have to be transformed to the stationary $\alpha \beta$ and rotating $d q$ reference-frames on the stator side and vice versa. This provides a powerful tool to create lumped parameter machinemodels such as the inverse- $\Gamma$ - and T-circuits; the latter shown in Fig. 2a. These are needed to synthesize current controllers and describe the machine operation during transients, faults, and in steady-state. The proposed method in the following sections is capable to accomplish this. In Section III-A rotor slots are interpreted analogously to the stator in a rotor 123 fundamental reference frame. Further, the Clarke transformation matrix of the HPD adapts to the rotor in Section III-B.

\section{A. The Rotor 123 Fundamental Reference Frame}

Similar to the introduction of a 123 fundamental reference frame on the stator side, such a reference frame may also be introduced on the rotor side. Here again, the current is considered for the sake of understanding. As for the stator, the rotor 123 fundamental reference frame represents the current distribution along the rotor circumference in $Q_{r}$ rotor slots. If the rotor is wound, an equivalent transformation from the practical $a b c$ rotor reference-frame to the rotor 123 fundamental reference frame as in $[3,(1)]$ is needed. Considering a squirrel-cage rotor, the described reference frame is identified by the subscript $123 r$.

Since it is recommended to let $Q_{r} \neq Q_{s}$ [7, p. 294] in induction machines (IMs) to avoid cogging, the number of harmonic planes obtained for the rotor differs from that of the stator. Therefore, the model of these harmonic planes has to be adapted.

\section{B. Clarke Transformation Matrix for Rotor Quantities}

To bring the rotor side quantities into the stator side harmonic planes, a Clarke transformation matrix for rotor quantities is introduced. Different to the stator side, currents are not forming coils by control. Therefore, we we do not assume half-wave symmetry. Moreover, we limit the transformation to the same harmonics as on the stator side, since all other harmonics cannot be excited [8], [9]. By substituting $m=Q_{r}$ in $[3,(2)]$, the rotor Clarke transformation matrix is presented in (4). Recall that $\xi$ denotes the biggest odd number, which is smaller than $m_{b}$. Moreover, another pre-factor is added. It accounts for the transformer characteristics of the IM and is derived in [7, (4.240)] with $N_{s}$ as the number of turns per coil in the stator.

$$
\begin{aligned}
& \mathbf{x}_{\alpha \beta}=\underbrace{2 N_{s}\left(\frac{m_{b}}{Q_{r}}\right)^{1-K} \cdot\left(\frac{2}{Q_{r}}\right)^{K} \mathbf{K}_{d} \mathbf{C}_{r}}_{\mathbf{T}_{123 r \rightarrow \alpha \beta}} \cdot \mathbf{x}_{123 r} \\
& \mathbf{x}_{123 r}=\underbrace{2 N_{s}\left(\frac{m_{b}}{Q_{r}}\right)^{K} \cdot\left(\frac{2}{Q_{r}}\right)^{1-K} \mathbf{C}_{r}^{\mathrm{T}} \mathbf{K}_{d}}_{\mathbf{T}_{\alpha \beta \rightarrow 123 r}} \cdot \mathbf{x}_{\alpha \beta} \\
& \mathbf{C}_{r}=\left[\begin{array}{ccccc}
1 & \cos (\vartheta) & \cos (2 \vartheta) & \ldots & \cos \left(\left(Q_{r}-1\right) \vartheta\right) \\
0 & \sin (\vartheta) & \sin (2 \vartheta) & \ldots & \sin \left(\left(Q_{r}-1\right) \vartheta\right) \\
1 & \cos (3 \vartheta) & \cos (6 \vartheta) & \ldots & \cos \left(\left(Q_{r}-1\right) 3 \vartheta\right) \\
0 & \sin (3 \vartheta) & \sin (6 \vartheta) & \ldots & \sin \left(\left(Q_{r}-1\right) 3 \vartheta\right) \\
\vdots & \vdots & \vdots & \ddots & \vdots \\
1 & \cos (\xi \vartheta) & \cos (2 \xi \vartheta) & \ldots & \cos \left(\left(Q_{r}-1\right) \xi \vartheta\right) \\
0 & \sin (\xi \vartheta) & \sin (2 \xi \vartheta) & \ldots & \sin \left(\left(Q_{r}-1\right) \xi \vartheta\right)
\end{array}\right] \\
& \vartheta=\delta+\theta_{r} \\
& \delta=\frac{\pi}{Q_{r}} \quad \theta_{r}(t)=\int_{t^{\prime}=0}^{t} \omega_{r}\left(t^{\prime}\right) d t^{\prime}+\theta_{r}(0)
\end{aligned}
$$

This transformation matrix is of size $m_{b} \times Q_{r}$ and is thus non-square. However, it can be derived from the DFT interpretation of the Clarke transformation. For even base cases, the rotor Clarke transformation matrix has to be expanded in accordance with (3).

Since the stator and rotor slots constitute the sampling points along an equally long sampling window of $360^{\circ}$ mechanically, 


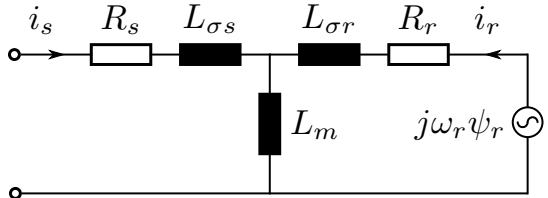

(a) Stator and rotor.

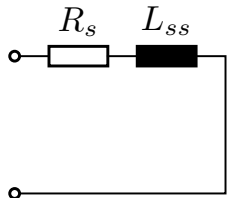

(b) Stator only.
Fig. 2. T-equivalent circuits (a) with rotor and (b) without rotor in vector spaces $\nu$ in a stator-oriented reference frame.

the sampling frequency of the DFT on the rotor side is lower than that on the stator side if $Q_{r}<Q_{s}$ and vice versa. Consequently, higher order frequency components pertain on the side having the most number of slots, according to the Nyquist-Shannon sampling theorem. In the HPD method, aliasing of rotor quantities and parameters can therefore appear if $Q_{r}<Q_{s}$. Hence, the same strategy as for the even base cases delivers a solution, as was explained in Section II-B and carried out in (3). Aliased frequencies correspond to the harmonic planes $h \geq\left\lfloor Q_{r} /\left(2 p_{b}\right)\right\rfloor$. The entries for these harmonic planes are replaced with zeros to avoid aliasing.

However, stator quantities need to be retained. Considering the equivalent T-model circuit of harmonic planes $h \geq$ $\left\lfloor Q_{r} /\left(2 p_{b}\right)\right\rfloor$ in Fig. 2b, it means that rotor quantities can be omitted from these planes due to their absence, whereas stator quantities are left unchanged. Thus, the model reduces to a series connection of the stator resistance $R_{s}$ and stator self-inductance $L_{s s}$ as shown in Fig. 2b. If the machine possesses more rotor than stator slots, $Q_{r}>Q_{s}$, the rotor side resolves more harmonics. In this case, the harmonic planes $h \geq\left\lfloor Q_{s} /\left(2 p_{b}\right)\right\rfloor$ are ignored. Since these do not contain stator side quantities, they cannot be excited.

\section{Simulation Results}

In this section, dynamic simulations using the HPD are conducted. To start with, Section IV-A showcases transformation results for an $m=3, p=2$ configuration using, on the one side, an odd base case and, on the other side, an even base case to demonstrate the need of separated cases. To conclude the HPD theory, Section IV-B compares transient and steady state behaviors of two machine models applying, on the one side, the VSD and, on the other side, the HPD. Concordantly, VSD and HPD transformations are applied to the dependent current variable in the respective cases.

The same machine as in [3] is considered. It is an $m=6$ phase machine with $p=1$ pole pair and $Q_{s}=36$ stator and $Q_{r}=28$ rotor slots. It is wound in YY30 configuration with one star point. When this machine is configured as an $m=3$ phase machine, it can be operated with $p=2$ pole pairs as well. The three possible phase-pole configurations are listed in Table I.

\section{A. Validation of Even Pole-Pair Cases}

As described in Section II-A, the current distribution of a configuration with an even number of pole pairs cannot be resolved with odd harmonics only. In [3], an odd base

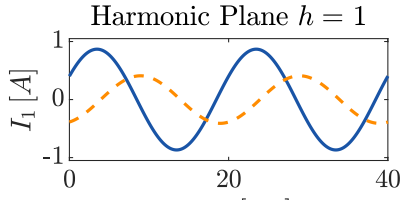

Time $[m s]$
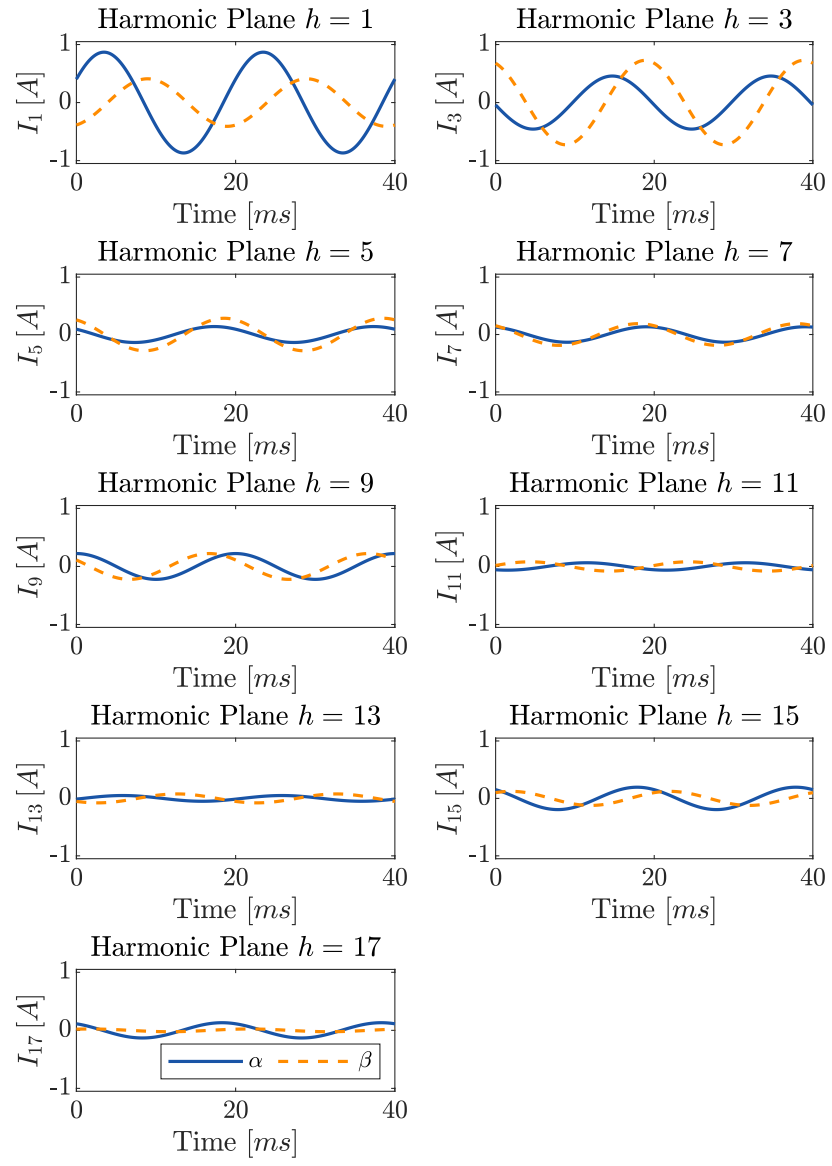

Fig. 3. $\alpha \beta$ reference-frame for the even configuration $m=3$, $p=2$ using the HPD with $m_{b, o}=18$, i.e. the odd base case. Blue solid lines indicate the $\alpha$ and yellow dashed lines indicate the $\beta$ component.

case with $m_{b, o}=18$ was introduced. If the HPD with this underlying odd base case is applied to the phase currents in (5), the $\alpha \beta$ reference-plane currents in Fig. 3 are obtained. The fundamental frequency is set to $\omega_{1}=100 \pi \mathrm{rad} / \mathrm{s}$, the current amplitude is set to $\hat{i}=1 \mathrm{~A}$ and $z \in\{a, b, c\}$ denotes the phase of the respective $a b c$ currents.

$$
\begin{aligned}
i_{z}(t) & =\hat{i} \cos \left(\omega_{1} t-\phi_{z}\right) \\
{\left[\begin{array}{lll}
\phi_{a} & \phi_{b} & \phi_{c}
\end{array}\right] } & =\left[\begin{array}{lll}
0 & \frac{2 \pi}{3} & \frac{4 \pi}{3}
\end{array}\right]
\end{aligned}
$$

It can be seen, that these results are not in accordance with the definition of an $\alpha \beta$ reference-frame:

- All harmonic planes are populated with non-zero components, which does not accord with the vector-space distribution.

TABLE I. CONFIGURATIONS OF THE YY30 MACHINE.

\begin{tabular}{c|c|c|c|c|c} 
& $m$ & $p$ & $Q_{s}$ & $q_{s}$ & \# Star Points \\
\hline Case 1 & 6 & 1 & 36 & 3 & 1 \\
Case 2 & 3 & 1 & 36 & 6 & 1 \\
Case 3 & 3 & 2 & 36 & 3 & 1
\end{tabular}


- The $\alpha$ - and $\beta$-components have different amplitudes in the same harmonic plane, which suggests an unbalance.

- The phase shifts between $\alpha$ - and $\beta$-components are not $90^{\circ}$ for all harmonic planes.

The derived expanded Clarke transformation matrix $\mathbf{T}_{123 \rightarrow \alpha \beta}$ is applied instead for the $m=3, p=2$ configuration. The vector-space distribution and the complex winding factors are listed in Table II. The fundamental component of the current distribution in vector space $\nu_{1}$ distributes over the harmonic planes $h \in\{1,5,7\}$. The fundamental equals a second harmonic in space-domain with respect to the mechanical angles. Due to the expansion of the even base case (comprising four vector-space planes and one axis) to the dimensions of the bigger odd base case (comprising nine vector-space planes), the higher order harmonic planes $h \in[11,17]$ are void, as predicted from the introduced zero entries in (3).

The $\alpha \beta$ reference-frame currents with the correctly applied HPD of this even base case are depicted in Fig. 4. It can be seen that the transformation delivers orthogonal $\alpha$ and $\beta$ currents in each harmonic plane. The amplitudes and phase shifts are in accordance to the complex winding factors that stem from the DFT of the current distribution as summarized in Table II. The sequences agree with the vector-space distribution provided in [3].

\section{B. Dynamic Comparison Between Harmonic Plane and Vector-Space Decomposition}

To demonstrate the equality between the HPD and VSD, transient simulations are conducted with stator-referenced models based on both types of transformations. For each harmonic plane, an inverse- $\Gamma$ model is used. VSD parameters are derived from the HPD ones as described by (6), which follows from conservation of energy. The magnetizing inductances of the inverse- $\Gamma$ model, $L_{M, \nu_{h}}^{\mathrm{VSD}}$, in the standard VSD framework are used as an example. $L_{M, h}^{\mathrm{HPD}}$ denotes the magnetizing inductance in the $h^{\text {th }}$ harmonic plane of the HPD model. All parameters undergo the same transformation. Note that only harmonic planes containing the same vector space are summed up.

$$
L_{M, \nu_{h}}^{\mathrm{VSD}}=\frac{m_{b}}{m} \sum_{h} L_{M, h}^{\mathrm{HPD}}\left|k_{d, h}\right|^{2} \quad \text { for }\left|\nu_{h}\right|=\text { const. }
$$

Both the HPD and VSD models are excited with an identical set of fundamental voltages such that the current in steady

TABLE II. Vector-Space Distribution AND Complex WINDING FACTOR FOR THE $m=3, p=2$ CONFIGURATION.

\begin{tabular}{c||c|c|c}
$\mathbf{h}$ & $\boldsymbol{\nu}_{h}$ & $\left|\mathbf{k}_{\mathbf{d}, \mathbf{h}}\right|$ & $\boldsymbol{\varphi}_{\boldsymbol{h}}$ \\
\hline \hline $\boldsymbol{\alpha} \boldsymbol{\beta}_{\mathbf{1}}$ & 1 & 0.960 & $20^{\circ}$ \\
\hline $\boldsymbol{\alpha} \boldsymbol{\beta}_{\mathbf{3}}$ & - & - & - \\
\hline $\boldsymbol{\alpha} \boldsymbol{\beta}_{\mathbf{5}}$ & -1 & 0.218 & $100^{\circ}$ \\
\hline $\boldsymbol{\alpha} \boldsymbol{\beta}_{\mathbf{7}}$ & 1 & 0.177 & $-40^{\circ}$ \\
\hline $\boldsymbol{\alpha} \boldsymbol{\beta}_{\mathbf{9}-\mathbf{1 7}}$ & - & - & -
\end{tabular}
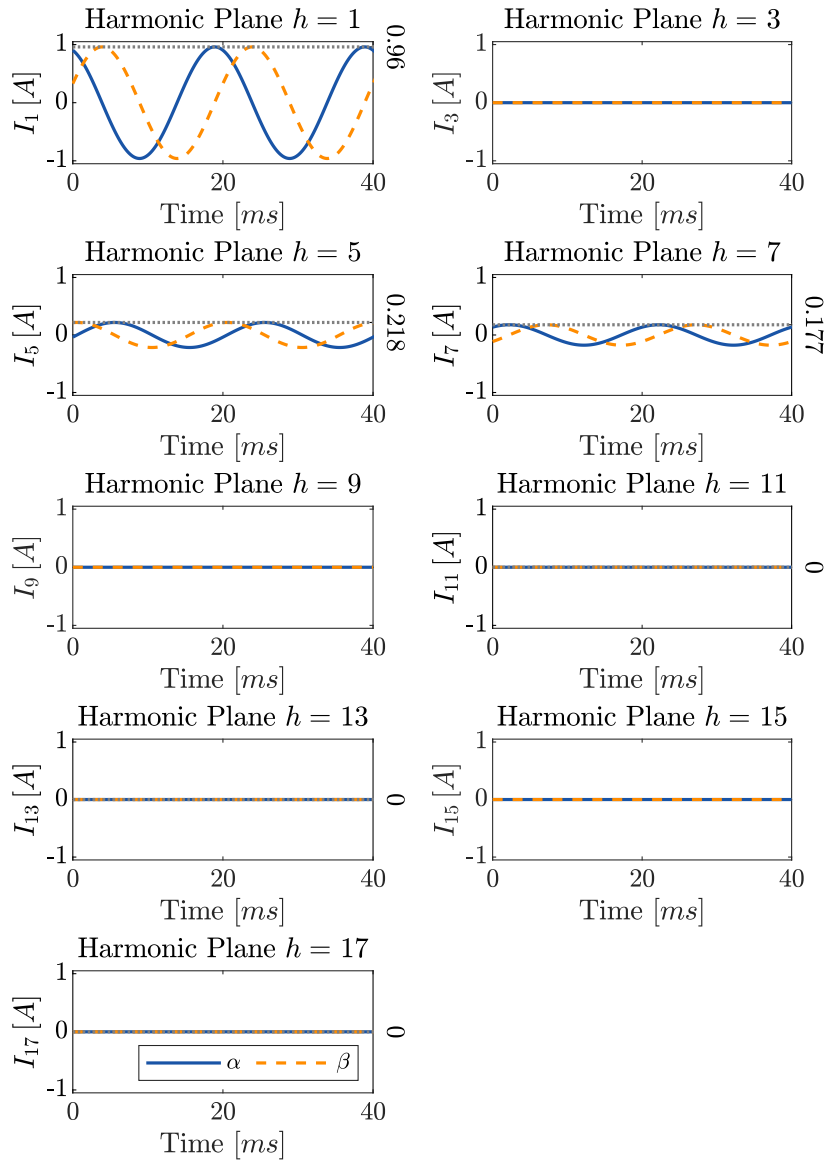

Fig. 4. $\alpha \beta$ reference-frame for the even configuration $m=3$, $p=2$ using the HPD with $m_{b, e}=9$, i.e. the even base case. Blue solid lines indicate the $\alpha$ and yellow dashed lines indicate the $\beta$ component.

state becomes $\hat{I}=1 \mathrm{p} . \mathrm{u}$. The electrical frequency is chosen to maintain the mechanical rotor speed at 0.95p.u. The resulting currents in phases A1 and A using the VSD model are displayed alongside the current in slot 2 (corresponding to the aforementioned phases) of the HPD model in Fig. 5. It shows that both models are equivalent in transient and steady state behavior. Additionally, the HPD model is capable of simulating a phase-pole reconfiguration, which is impractical with a VSD model. For instance, when the machine transitions from a $m=6, p=1$ to a $m=3, p=2$ configuration, the resulting current in slot 2 looks as in Fig. 6.

\section{OUTLOOK}

With the completed HPD in this paper, we lay the foundations for further investigations of multiphase electrical machines. The main application area for the HPD is expected to be variable phase-pole machines, e.g. the ISCAD [5], WICSC machine [4], [6]. With the unified framework of all phase-pole configurations achieved by the HPD, we prepare a seamless control of the transition between two phase-pole configurations. 

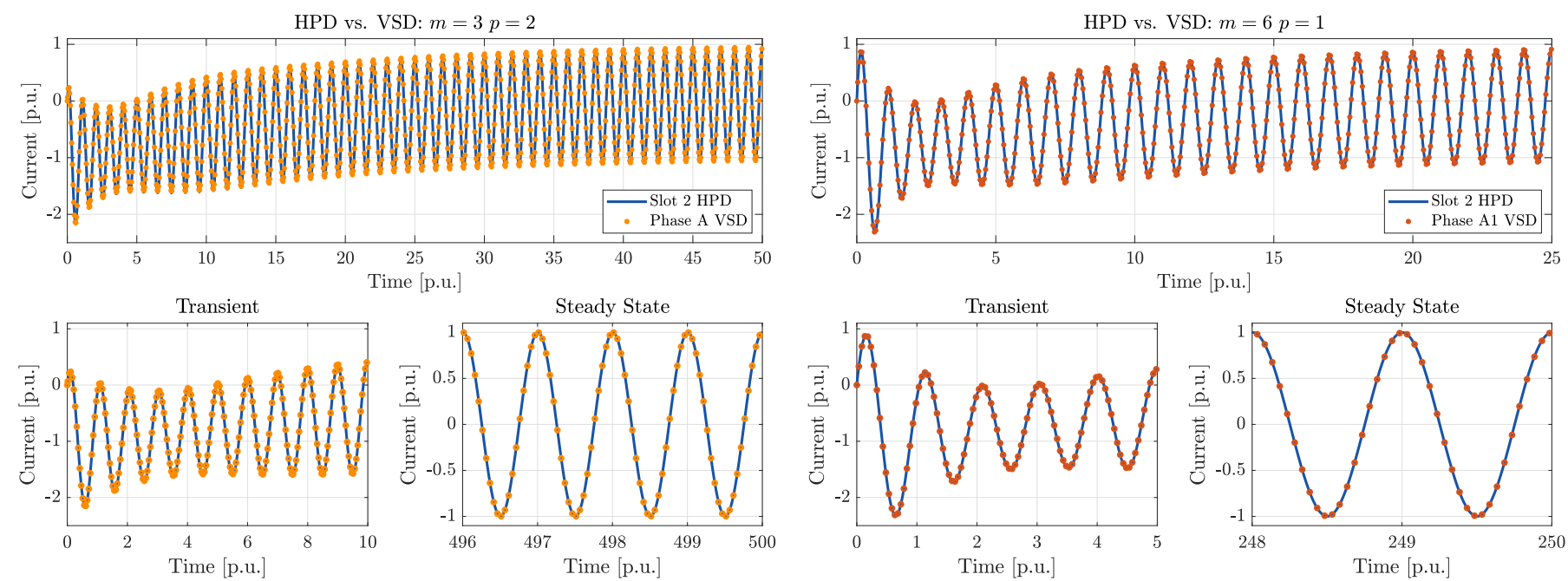

(a) $m=3, p=2$ Phase A

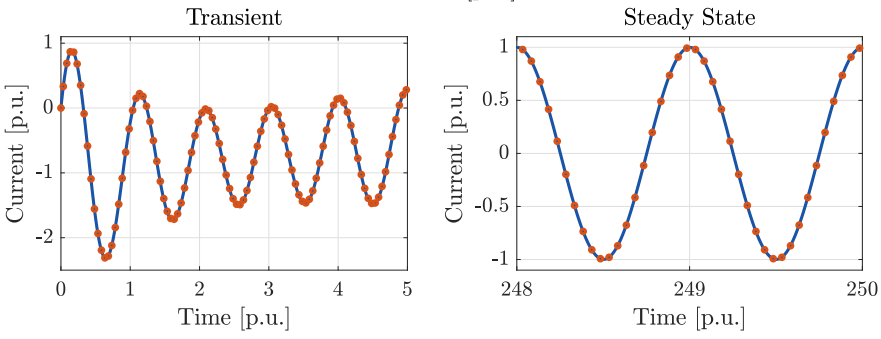

(b) $m=6, p=1$ Phase A1

Fig. 5. Dynamic current comparison between HPD, blue and solid line, and VSD, yellow and orange dots.

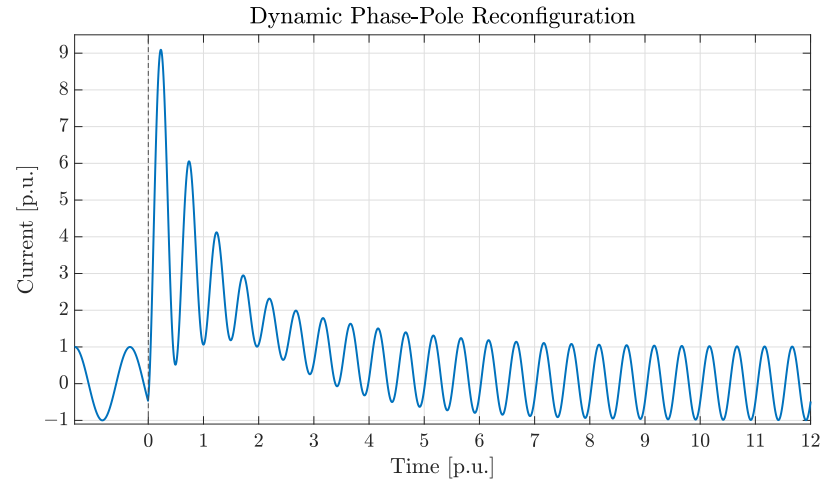

Fig. 6. Current in Phase A1 and A (slot 2) during the reconfiguration from $m=6, p=1$ to $m=3, p=2$. The configuration is switched at $t=0$ p.u.

\section{CONCLUSION}

This paper completes the theory of a harmonic plane decomposition (HPD) introduced in the companion paper [3]. The lack of half-wave symmetry in configurations with an even number of pole pairs and an inherent mismatch in the number of rotor and stator slots lead to potential aliasing in the inverse Clarke transformation. These aliasing effects are independent from each other and can appear simultaneously. For this reason, the Clarke transformation matrix has to be adapted. For an even number of pole pairs, adapted base cases and Clarke matrices are defined by expanding them to maintain the same dimension of the matrices used in the odd base case. Similarly, the Clarke matrix is adapted for rotor quantities to enable transformations between the rotor and stator sides. With these adaptions, it is possible to model each harmonic plane, $h$, by conventional induction machine (IM) models, such as the inverse- $\Gamma$ and $\mathrm{T}$-model for all phase-pole configurations.

The contribution from both papers is expected to facilitate the modeling and control of multiphase machines, in particular those with reconfigurable and independently controlled stator windings.

\section{REFERENCES}

[1] Y. Zhao and T. A. Lipo, "Space vector PWM control of dual three-phase induction machine using vector space decomposition," IEEE Transactions on Industry Applications, vol. 31, no. 5, pp. 1100-1109, 1995.

[2] E. Levi, R. Bojoi, F. Profumo, H. A. Toliyat, and S. Williamson, "Multiphase induction motor drives - a technology status review," IET Electric Power Applications, vol. 1, no. 4, pp. 489-516, 2007.

[3] Y. Wu, G. F. Olson, L. Peretti, and O. Wallmark, "An extension of the vector-space decomposition for variable phase-pole electrical machines part I," in IECON 2020 - 46th Annual Conference of the IEEE Industrial Electronics Society, Conference Proceedings.

[4] K. Bitsi, O. Wallmark, and S. Bosga, "An induction machine with wound independently-controlled stator coils," in 2019 22nd International Conference on Electrical Machines and Systems (ICEMS), Conference Proceedings, pp. 1-5.

[5] G. Dajaku and D. Gerling, "Low costs and high efficiency asynchronous machine with stator cage winding," in 2014 IEEE International Electric Vehicle Conference (IEVC), Conference Proceedings, pp. 1-6.

[6] E. Libbos, B. Ku, S. Agrawal, S. Tungare, A. Banerjee, and P. T. Krein, "Loss minimization and maximum torque per ampere operation for variable-pole induction machines," IEEE Transactions on Transportation Electrification, pp. 1-1, 2020.

[7] T. A. Lipo, Introduction to AC Machine Design. Hoboken, NJ, USA: John Wiley \& Sons, Inc., 2017.

[8] M. P. Magill and P. T. Krein, "A dynamic pole-phase modulation induction machine model," in 2015 IEEE International Electric Machines Drives Conference (IEMDC), Conference Proceedings, pp. 13-19.

[9] H. R. Fudeh and C. M. Ong, "Modeling and analysis of induction machines containing space harmonics part i: Modeling and transformation," IEEE Transactions on Power Apparatus and Systems, vol. PAS-102, no. 8 , pp. 2608-2615, 1983. 